\title{
Estudo da Modificação Química de Polidienos do Tipo SBR e BR
}

\author{
Tatiana L. A. C. Rocha, Robert H. Schuster \\ Deutsches Institut für Kautschuktechnologie e.V. \\ Marly M. Jacobi, Dimitrios Samios \\ Instituto de Química, UFRGS
}

Resumo: A utilização de modificações químicas de polidienos comerciais tem sido estudada como um meio alternativo à síntese de novos polímeros, para otimização das propriedades finais destes materiais através da introdução de diferentes grupamentos reativos na cadeia polimérica. A modificação química pode ser feita através de diferentes métodos, os quais podem ser realizados tanto em solução como em massa, onde podem ser destacadas as reações de epoxidação, sulfonação, maleinização, carboxilação, etc. Neste trabalho foi estudado o método de epoxidação de borrachas do tipo SBR e BR. Foi possível observar que mesmo pequenos graus de modificação química causam mudanças marcantes nas propriedades finais dos polímeros, como determinado para a temperatura de transição vítrea.

Palavras-chave: Modificação química, polidienos, $S B R, B R$.

\section{Study of Chemical Modification of SBR and BR Polydiene}

Abstract: Chemical modification of polydiene has been studied as an alternative route to obtain modified polymers with improved final properties. This improvement is due to the introduction of different kinds of reactive groups into a polymer chain, and it can be done in solution as well as in bulk. The chemical modification can be carried out by different methods such as epoxidation, maleination, carboxylation, sulfonation etc. In this work we show that in the epoxidation of SBR and BR even a small degree of modification can change the final properties of the polymer, as it occurred for the glass transition temperature.

Keywords: Chemical modification, polydiene, $S B R, B R$.

\section{Introdução}

O estudo a respeito de modificações químicas de polidienos tem demonstrado ser de grande interesse devido a possibilidade de otimização das propriedades físicas e mecânicas dos mesmos, através da introdução de grupamentos reativos na cadeia polimérica. A modificação química de polidienos tem possibilitado muito bons resultados no que se refere à melhoria de propriedades, como por exemplo: resistência a óleos e solventes, bem como melhoria da resistência a permeabilidade a gases, melhor aderência a materiais cerâmicos, metálicos e têxteis e ainda melhoria na propriedade de adesão ao piso úmido (wet-skid) ${ }^{[1]}$.

Diferentes tipos de reação para introdução de grupamentos reativos na cadeia polimérica podem ser utilizados para a modificação química de polidienos, dentre os quais podem ser destacadas as reações de sulfonação, maleinização e epoxidação ${ }^{[2-6]}$.

A epoxidação de polidienos tem sido cada vez mais estudada devido ao crescente interesse comercial na área da borracha natural (NR) e de algumas borrachas sintéticas (IR, BR, etc $)^{[3,4]}$, bem como na produção de microgéis ${ }^{[7]}$. Um dos métodos mais amplamente empregados para epoxidação de polidienos é a reação de peróxido de hidrogênio com ácido fórmico, formando o perácido in situ que reagirá com a dupla ligação ${ }^{[1,3]}$. Essa reação pode ser influenciada por vários fatores como por exemplo, microestrutura do polímero ${ }^{[8]}$, temperatura e concentração da solução ${ }^{[1,3,4]}$.

Neste trabalho borrachas do tipo BR e SBR foram modificadas quimicamente através da reação de epoxidação e a influência desta modificação sobre as propriedades finais das borrachas foi estudada.

\section{Experimental}

Os polímeros utilizados neste trabalho (Tabela 1) são borrachas especiais do tipo polibutadieno, BR, e copolímeros aleatórios de butadieno e estireno, SBR, obtidos em solução, e fornecidos pela empresa alemã Bayer (AG). 
Tabela 1. Característica dos polímeros utilizados neste trabalho

\begin{tabular}{|c|c|c|c|c|c|c|c|}
\hline $\begin{array}{l}\text { Borrachas } \\
\text { Nome } \\
\text { Comercial }\end{array}$ & Borrachas & Mw & $\mathbf{M n} / \mathbf{M w}$ & $\begin{array}{c}\text { Estireno } \\
(\text { massa\%) }\end{array}$ & $\begin{array}{c}\text { Unidades 1,2 } \\
(\operatorname{massa} \%)\end{array}$ & $\begin{array}{c}\text { 1,4-cis } \\
(\operatorname{massa} \%)\end{array}$ & $\begin{array}{c}\text { 1,4-trans } \\
(\text { massa \%) }\end{array}$ \\
\hline SL25-0 & SBR-10 & 172650 & - & 25 & 8 & & \\
\hline VSL2525-0 & SBR-32 & 206600 & 2,12 & 25 & 25 & & \\
\hline VSL5525-0 & SBR-73 & 188600 & - & 25 & 50 & & \\
\hline GBR223U & SBR & 212400 & 1,20 & 25 & 24 & & \\
\hline GBR252H & BR1 & 137000 & 1,08 & - & 10 & 40 & 50 \\
\hline GBR252Q & BR2 & 265000 & 1,03 & - & 10 & 40 & 50 \\
\hline
\end{tabular}

\section{Epoxidação}

A borracha foi dissolvida em tolueno $(1 \mathrm{~g}$ polímero $/ 15 \mathrm{~mL}$ solvente) e após a dissolução total foi adicionado o surfactante, polietilenoglicol-sorbitan-monolaurato (Tween20) a 1,9×10-4 \% em massa. O sistema foi aquecido até a temperatura desejada e foi adicionada a quantidade molar de ácido fórmico e peróxido de hidrogênio. Ao final da reação a solução da borracha modificada foi neutralizada com solução de $\mathrm{Na}_{2} \mathrm{CO}_{3}(5 \%)$, lavada com água destilada. A fase orgânica foi separada da fase aquosa, novamente lavada até que não fosse mais detectada a presença de peróxido, precipitada em etanol e a borracha epoxidada foi seca sob vácuo até atingir peso constante.

\section{Caracterização dos polímeros modificados}

O teor de epoxidação foi obtido através da técnica de Ressonância magnética nuclear de hidrogênio (RMN de $\left.{ }^{1} \mathrm{H}\right)$ em um equipamento da firma Bruker modelo DRX 500.

As modificações químicas e de reações secundárias foram monitoradas através de espectroscopia de infravermelho com transformada de Fourier (FT-IR). A partir de uma solução da borracha modificada foram obtidos filmes sobre placas de $\mathrm{NaCl}, \mathrm{KBr}$ ou $\mathrm{BaF}_{2}$, os quais foram submetidos à análise em um equipamento FT-IR modelo Nicolet 60-Nexus com resolução de 32 scans e $4 \mathrm{~cm}^{-1}$.

O comportamento térmico dos polímeros modificados foi estudado através da técnica de calorimetria exploratória diferencial (Modulated DSC 2920-TA Instruments) sendo utilizada uma taxa de aquecimento de $10{ }^{\circ} \mathrm{C} /$ minuto sob atmosfera de $\mathrm{N}_{2}$ em cápsulas herméticas. As análises de termogravimetria diferencial (TG/DTG) foram conduzidas em balança termogravimétrica (Hi-Res TGA 2950 Thermogravimetric analyzer-TA Instruments), com taxa de aquecimento de $10{ }^{\circ} \mathrm{C} /$ minuto, sob atmosfera de $\mathrm{N}_{2}$, a partir da temperatura ambiente até $500{ }^{\circ} \mathrm{C}$ e, sob atmosfera oxidativa, de $500-750{ }^{\circ} \mathrm{C}$.

\section{Resultados e Discussão}

\section{Epoxidação}

Segundo discutido na literatura ${ }^{[9]}$ a reação de epoxidação ocorre conforme o mecanismo proposto na Figura 1, o qual

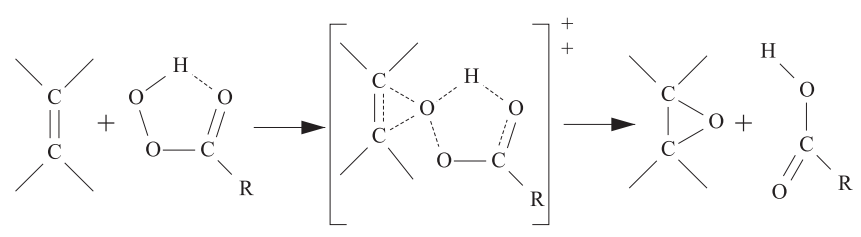

Figura 1. Representação esquemática provável mecanismo da reação de epoxidação ${ }^{[9]}$

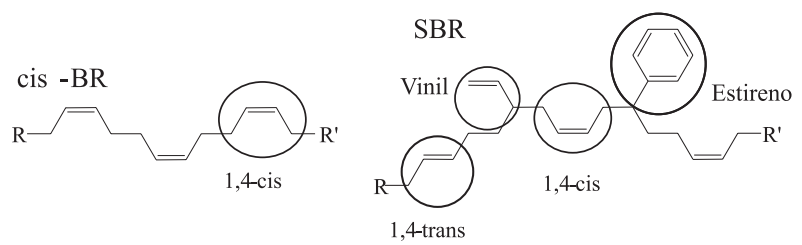

Figura 2. Estrutura geral das moléculas de borrachas do tipo SBR e BR

sugere que a formação da oxirana (anel epóxido) envolve um processo cíclico polar onde o próton é transferido intramolecularmente para o oxigênio carbonílico com ataque simultâneo da ligação $\pi$ do alceno. Esse mecanismo também é conhecido como mecanismo "Butterfly".

A velocidade da reação de epoxidação é dependente da presença dos diferentes tipos de ligações (vinila, 1,4-cis e 1,4trans) presente na molécula polimérica. Na Figura 2 são apresentadas de forma geral a estrutura das borrachas utilizadas neste estudo.

\section{Infravermelho}

$\mathrm{O}$ aparecimento de bandas na região em torno de 3500 $\mathrm{cm}^{1}$, relativas a reações secundárias resultantes a partir da reação do grupamento epóxi com o ácido regenerado durante a reação não foi observado. Por este motivo, na Figura 3, é apresentado apenas o espectro típico da região de interesse de uma borracha do tipo SBR antes e após a epoxidação.

A análise do espectro para SBR-32 da Figura 3 mostra a banda relativa ao estiramento fora do plano para o estireno a $700 \mathrm{~cm}^{-1} \mathrm{e}$ as bandas características dos grupamentos 1,4-cisbutadieno $\left(760 \mathrm{~cm}^{1}\right)$ e 1,4-trans butadieno $\left(960 \mathrm{~cm}^{-1}\right)$ e unidades 1,2 butadieno $\left(911 \mathrm{~cm}^{-1}\right)$. No espectro para a SBR-32 (30\%epóxi) foi observado o aparecimento de uma nova banda na região de $740 \mathrm{~cm}^{-1}$, do espectro relacionado à vibração do grupamento cis-epóxido. Na região de $760 \mathrm{~cm}^{-1}$ deve estar 


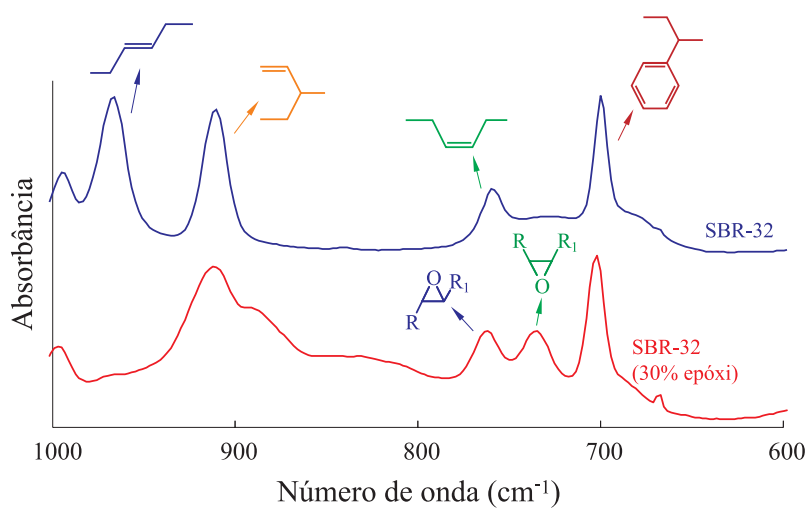

Figura 3. Espectro de FT-IR da região de análise característica dos grupamentos epóxi para SBR-32 não epoxidada e SBR-32 epoxidada.

presente a banda relativa ao grupamento trans-epóxido, que aparece também como um ombro no pico da região de 890 $\mathrm{cm}^{-1}$. Considerando a sobreposição que ocorre entre as bandas dos grupamentos trans-epóxido e 1,4-cis butadieno, é difícil a quantificação dos grupamentos epóxidos através da espectroscopia de infravermelho. Porém, foi observado que a banda relativa ao grupamento 1,4-trans butadieno praticamente desaparece para a SBR-32 (30\% epóxi) indicando que essas ligações reagiram, possibilitando uma análise qualitativa da presença dos grupamentos epóxidos.

\section{$R M N$ de ${ }^{1} \mathrm{H}$}

Quantitativamente foi possível o monitoramento da reação de epoxidação através da espectroscopia de RMN de ${ }^{1} \mathrm{H}$, onde para a determinação do grau de epoxidação foram consideradas as áreas normalizadas dos sinais dos hidrogênios dos grupamentos relativos a unidades dos grupamentos estireno, 1,4-cis butadieno, 1,4-trans butadieno e 1,2 butadieno antes e após a epoxidação como mostra a Figura 4.

$\mathrm{Na}$ Figura 4 pode ser observado um aumento da área dos sinais relativos aos hidrogênios dos grupamentos trans-epóxido $(\delta=2,45 \mathrm{ppm})$ e cis-epóxido $(\delta=2,70 \mathrm{ppm})$ ao mesmo tempo que há uma diminuição na área dos sinais dos grupamentos 1,4-cis e 1,4-trans butadieno $(\delta=5,40 \mathrm{ppm})$. Além disso a área do sinal associado aos dois hidrogênios terminais da unidade 1,2 butadieno $(\delta=4,9 \mathrm{ppm})$ permanece praticamente constante. Apenas para graus muito altos de epoxidação foi percebida uma variação desse sinal. Baseado nos espectros apresentados na Figura 4 a) e b) foi observado que à medida que a epoxidação ocorre, as unidades 1,4-cis são mais rapidamente consumidas que as unidades 1,4-trans e o sinal relativo ao grupamento epóxido cis $(\delta=2,90 \mathrm{ppm})$ aparece maior que o sinal do grupamento epóxido trans $(\delta=2,67 \mathrm{ppm})$. Com isto e baseados em estudos anteriores ${ }^{[8]}$ foi possível comprovar que as unidades 1,4-cis e trans-butadieno são consumidas com uma velocidade maior do que as unidades butadieno 1,2 devido a sua maior reatividade. A presença dessas unidades ( 1,2 butadieno) menos reativas influencia fortemente o comportamento da reação de epoxidação como pode ser visto na Figura 5.

$\mathrm{Na}$ Figura $5 \mathrm{o}$ teor de epoxidação aumenta à medida que o teor de unidades 1,2 butadieno diminui. Isto indica que estas

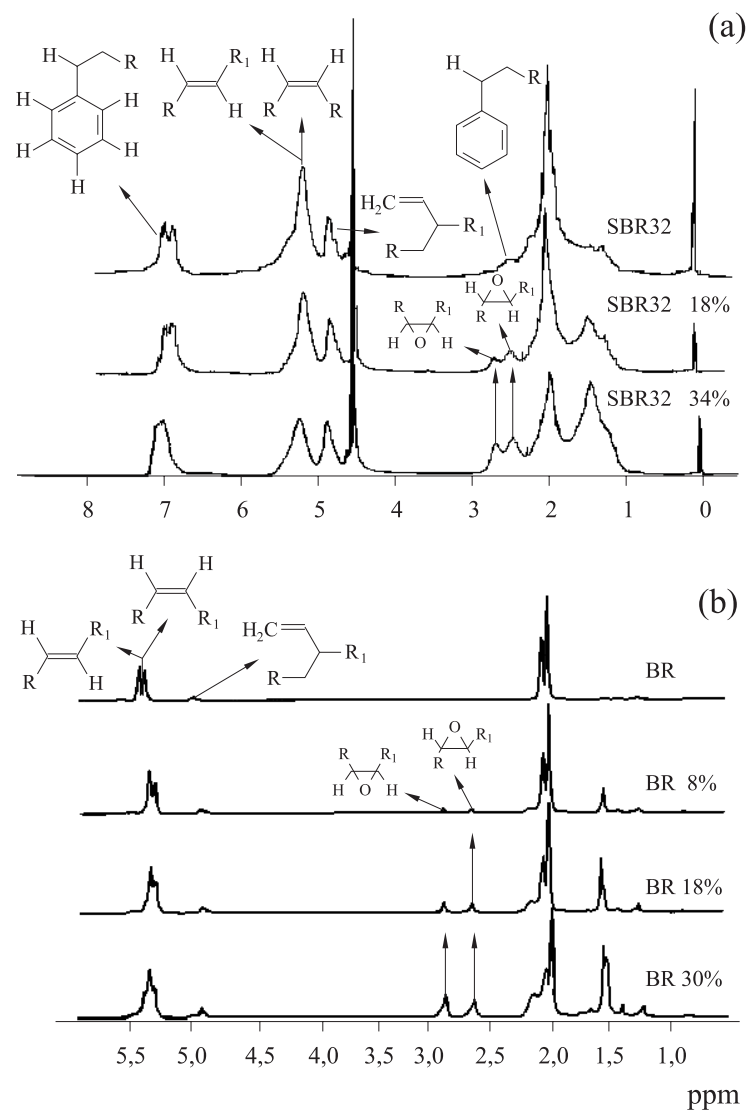

Figura 4. Espectro de ${ }^{1} \mathrm{H}-\mathrm{NMR}$ comparativo para a) SBR-32 e b) para BR1 não epoxidada, e para diferentes graus de epoxidação.

unidades são menos reativas que as unidades 1,4-cis e 1,4 trans butadieno. Pode ser observado também que, no início da reação, há um rápido aumento da conversão das ligações duplas em grupamentos epóxido e com o decorrer da reação há uma diminuição na velocidade de conversão dessas ligações tendendo a um valor limite. Isto ocorre porque com o decorrer da reação há um decréscimo da concentração de ligações duplas, bem como um decréscimo da concentração de peróxido de hidrogênio no meio reacional. Além disso, deve ser considerada a existência de três diferentes tipos de unidades butadiênicas (1,4-cis, 1,4-trans e 1,2 butadieno) e com o decorrer da reação são introduzidos grupamentos epóxido na cadeia que causam um efeito indutivo, diminuin-

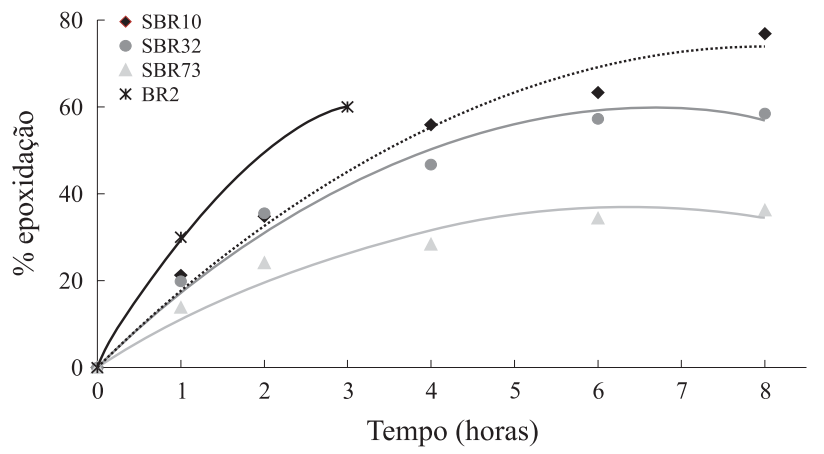

Figura 5. Influência do teor de unidades 1,2-butadieno sobre a velocidade de epoxidação de borrachas do tipo SBR. 


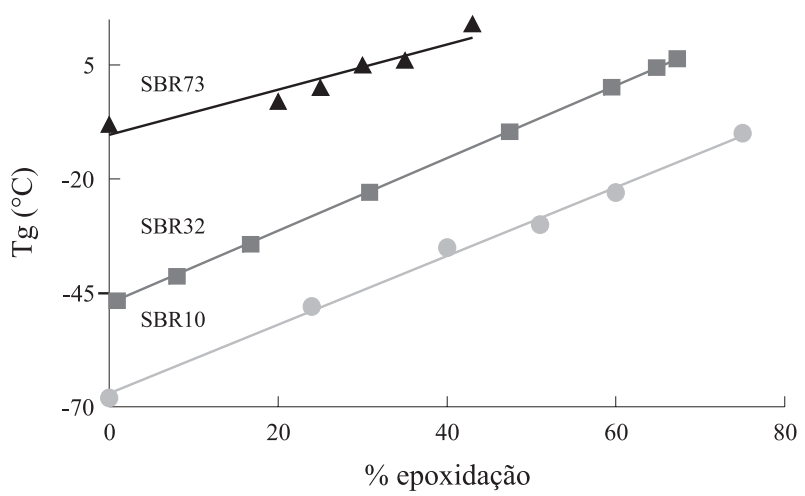

Figura 6. Influência do grau de epoxidação sobre a Tg para SBR com teores variáveis de unidades 1,2-butadieno.

do a velocidade de conversão das ligações duplas. Essas diferentes unidades butadiênicas apresentam diferentes velocidades de reação. À medida que a reação de epoxidação ocorre, mais unidades 1,4 butadieno vão sendo consumidas e o sistema passa a ter mais unidades 1,2 butadieno que reagem mais lentamente, levando a tendência de ser atingido um valor limite do grau de epoxidação.

Analisando as borrachas SBR10 e BR2, ambas com teor de unidades vinílicas próximo a $10 \mathrm{~mol} \%$, pode ser observado que a BR2 é epoxidada mais rapidamente do que a SBR10. Isso ocorre devido a presença dos grupamentos estirênicos na SBR10, os quais dificultam a reação de epoxidação levando a uma velocidade de reação mais lenta.

A epoxidação leva a um aumento na interação entre as cadeias poliméricas, causado pelo aumento da polaridade das mesmas. Como conseqüência, ocorre uma modificação na mobilidade das cadeias o que é refletida na temperatura de transição vítrea (Tg) do material. A Figura 6 apresenta a variação da Tg com o grau de epoxidação para as borrachas SBR's com diferentes teores de unidades 1,2 butadieno.

Na Figura 6 é observado que com o aumento do grau de epoxidação há um aumento linear na temperatura de transição vítrea $(\mathrm{Tg})$. A taxa de aumento da $\mathrm{Tg}$ determinada para as borrachas estudadas foi de $0,73^{\circ} \mathrm{C} / \mathrm{mol} \%$ para SBR-10, $0,80^{\circ} \mathrm{C} / \mathrm{mol} \%$ para SBR-32 e de $0,54^{\circ} \mathrm{C} / \mathrm{mol} \%$ para SBR- 73 , onde foi observado que a borracha com maior teor de unidade 1,2 butadieno apresenta a menor taxa de variação da $\mathrm{Tg}$, devido a menor reatividade dessas unidades. Um comportamento similar foi encontrado para as borrachas do tipo BR1 e BR2.

À medida que o grau de epoxidação aumenta há um aumento do momento de dipolo entre duas cadeias levando a uma interação eletromagnética mais forte para as cadeias epoxidadas do que para as não epoxidadas, resultando em uma dissipação de energia que contribui para o movimento de fricção molecular, causando um aumento da Tg. Ou seja, quanto maior o teor de epoxidação, maior será a fricção entre as cadeias e, maior será a energia necessária para que essas cadeias se movimentem, provocando um aumento da $\mathrm{Tg}$.

\section{Conclusões}

O grau de modificação química de um polímero é fortemente influenciado pela microestrutura do mesmo. Foi possível a obtenção de polímeros com baixo grau de modificação e boa reprodutibilidade dos resultados. Mesmo pequenos graus de modificação química apresentam uma influência bastante marcante nas propriedades finais dos mesmos, como por exemplo o aumento da temperatura de transição vítrea.

\section{Agradecimentos}

Os autores gostariam de agradecer à empresa Bayer AG pela disponibilização das borrachas, ao CNPq e DAAD pelo apoio financeiro e ao Professor Hahn da Universidade de Colônia (Alemanha) pelas medidas de ${ }^{1} \mathrm{H}-\mathrm{RMN}$.

\section{Referências Bibliográficas}

1 Gupta, B. R. \& De, S. K. - "Elastomer Technology Handbook", N. P. Cheremisinoff (volume editor) (1993)

2 Schuster, R. H. -Angew. Makromol. Chemie 202/203 p.159 (1992)

3 Zuchowska, D. -Polymer, 21, p.514 (1980)

4 Baker, C. S. L.; Gelling, I. R. e Newell, R.-Rubber Chemistry and Technology 58, p.67 (1985)

5 Sheng, J;. X. L. Lu e K. d. Yao; J. Macromol. Sci.Chem.A27(2) (1990) 167

6 Mokrini, A. e Acosta, J. L.; Polymer 42 (2001) 9

7 Hallensleben, M. L.; Schmidt, H. R. e Schuster, R. H.; Die Angewandte Makromolekulare Chemie, 227, p.87 (1995)

8 Rocha, T. L. A. C.; Jacobi, M. M.; Neto, C. P.; Schneider, C. G. e Schuster, R. H.; Kautschuk Gummi Kunstoffe, 11, p.590(2002)

9.. Bartlett, P. D; Rec. Chem. Progr. 11 (1950) 47

Recebido: 27/02/04

Reenviado: 05/07/04

Aprovado: 13/08/04 